\title{
Discriminative learning of similar objects enhances memory for the objects and contexts
}

\author{
Wenxi Zhou, Haoyu Chen, and liongjiong Yang \\ School of Psychological and Cognitive Sciences and Beijing Key Laboratory of Behavior and Mental Health, Peking University, Beijing, \\ 100871, China
}

\begin{abstract}
How to improve our episodic memory is an important issue in the field of memory. In the present study, we used a discriminative learning paradigm that was similar to a paradigm used in animal studies. In Experiment 1, a picture (e.g., a dog) was either paired with an identical picture, with a similar picture of the same concept (e.g., another dog), or with a picture of a different concept (e.g., a cat). Then, after intervals of $10 \mathrm{~min}, 1 \mathrm{~d}$, and $1 \mathrm{wk}$, participants were asked to perform a 2-alternative forcedchoice (2AFC) task to discriminate between a repeated and a similar picture, followed by the contextual judgment. In Experiment 2, eye movements were measured when participants encoded the pairs of pictures. The results showed that by discriminative learning, there was better memory performance in the 2AFC task for the "same" and "similar" conditions than for the "different" condition. In addition, there was better contextual memory performance for the "similar" condition than for the other two conditions. With regard to the eye movements, the participants were more likely to fixate on the lure objects and made more saccades between the target and lure objects in the "similar" (versus "different") condition. The number of saccades predicted how well the targets were remembered in both the 2AFC and contextual memory tasks. These results suggested that with discriminative learning of similar objects, detailed information could be better encoded by distinguishing the object from similar interferences, making the details and the contexts better remembered and retained over time.
\end{abstract}

One important feature of episodic memory is that it contains detailed and vivid representations. Remembering and maintaining detailed information is important for our daily lives. Using this form of memory, we can identify whether we have met a specific person before and quickly detect any changes in the person. We can also distinguish which type of ice cream we like best from our options. However, detailed information is more difficult to remember than general/gist information (Moscovitch et al. 2016). Patients with memory impairments have great difficulty learning stimulus details, such as the color, position or time of a stimulus, even when they sometimes can recognize the learned stimulus individually (Mayes et al. 2007).

Improving episodic memory, especially memory involving detailed information over time, is an important issue in the field of memory (Moscovitch et al. 2016). Memory can be enhanced by manipulating the encoding, consolidation and retrieval processes. From among the available methods, a paradigm of discriminative learning used in animal studies has been shown to facilitate fear conditioning significantly (Frankland et al. 1998; McHugh et al. 2007; Wang et al. 2009; Sahay et al. 2011; Niibori et al. 2012; Czerniawski and Guzowski 2014). For example, in a study by Wang et al. (2009), mice were trained for $3 \mathrm{~d}$ in context A (paired with shock) and context B (never paired with shock, but similar to context $\mathrm{A}$ ). When they acquired the fear conditioning, the mice froze more frequently in context $A$ than in context $B$, and this pattern persisted for $42 \mathrm{~d}$. The discriminative fear learning is different from contextual fear conditioning. In the contextual fear conditioning paradigm, although rats could distinguish between a training cage and a control cage, they manifested generalization $1 \mathrm{mo}$ later, exhibiting similar freezing behavior in both the training and control cages (Wiltgen et al. 2010). Moreover, animals with damage to the hippocampus could discriminate between similar contexts in certain conditions (e.g., Good and Honey 1991; Frankland et al. 1998; McHugh et al. 2007). For example, after

\section{Corresponding author: yangij@pku.edu.cn}

Article is online at http://www.learnmem.org/cgi/doi/10.1101//m.047514.118. mice were overtrained to discriminate between two similar contexts, control and dentate-mutant mice displayed similar freezing patterns in contexts A and B (McHugh et al. 2007). These findings suggest that discriminative learning is an efficient approach to facilitate learning and retain detailed information. The discriminative learning of similar stimuli is thus regarded as a paradigm for testing pattern separation, i.e., the ability to form distinct representations from similar stimuli (McClelland et al. 1995).

Whether the discriminative learning of similar stimuli enhances memory performance in human subjects is unknown. To our knowledge, there are no comparable studies that have asked subjects to compare similar stimuli when they are simultaneously presented during encoding. Nevertheless, some indirect evidence suggests that there may be similar mechanisms involved in facilitating memory across both animals and humans. For example, when stimuli were presented continuously, memory performance was better for blocked than interleaved presentations (Carvalho and Goldstone 2014). This finding suggested that studying each concept in separate blocks emphasizes the similarities within each category, thus increasing within-category comparisons. In addition, Koutstaal et al. (1999) showed that when subjects were asked to pay attention to specific parts of perceptual features of an object during encoding, aging people reduced gist-based false recognition during old/new recognition, which suggested that distinctive features of each picture are efficiently processed. Therefore, it is possible that memory can be enhanced when two similar objects from the same concept are presented simultaneously, compared to when two objects from different concepts are presented. This study was designed to test this hypothesis in both behavioral and eye-movement experiments.

(C) 2018 Zhou et al. This article is distributed exclusively by Cold Spring Harbor Laboratory Press for the first 12 months after the full-issue publication date (see http://learnmem.cshlp.org/site/misc/terms.xhtml). After 12 months, it is available under a Creative Commons License (Attribution-NonCommercial 4.0 International), as described at http://creativecommons.org/licenses/by-nc/4.0/. 
In the present study, two pictures were presented simultaneously during encoding, and three encoding conditions were manipulated. In the "similar" condition, a picture was paired with a similar picture from the same concept (e.g., two similar apples). In the "different" condition, a picture was paired with a picture from a different concept (e.g., an apple and a peach). In the "same" condition, a picture was paired with an identical picture (e.g., two identical apples). We defined critical "discriminative learning" as learning a picture under the "similar" condition. By asking participants to discriminate between similar pictures, the detailed and perceptual difference would be more elaboratively encoded. In contrast, in the "different" condition, discriminating between the pictures requires conceptual/semantic rather than perceptually detailed information. In the "same" condition, participants have to compare every detail to ensure that they are the same pictures, which is similar to the "similar" condition in this sense.

We explored the effect of discriminative learning in two respects. First, we asked whether the discriminative encoding of similar objects enhanced different types of memory, i.e., item memory and relational/contextual memory (Cohen and Eichenbaum 1993; Eichenbaum et al. 1994; Hockley and Consoli 1999; Davachi et al. 2003; Buchler et al. 2008). The two types of memory rely on distinct cognitive and neural mechanisms (Eichenbaum et al. 2007; Mayes et al. 2007). The 2-alternative forced-choice (2AFC) task has been proven to be an efficient way to test item memory of pictures (e.g., Brady et al. 2008; Huebner and Gegenfurtner 2012; Andermane and Bowers 2015). For example, in a study by Brady et al. (2008), after participants viewed pictures of 2500 objects, they made a $2 A F C$ judgment 10 min later. One of the old objects was paired with an object from a different concept, a different object from the same concept, or the same object in a different state. The results showed that the 2AFC performance was high in all three conditions $(92 \%, 88 \%$, and $87 \%)$. Among them, when participants could distinguish an old object from a lure object in the same concept, the details of the object were remembered even after $1 \mathrm{wk}$ (Andermane and Bowers 2015). In the present study, the 2AFC task was used to test the item memory at different retention intervals.

Unlike item-specific memory, relational or contextual memory refers to memory for relationships between individual items or between the item and its context (e.g., the task performed) (Cohen and Eichenbaum 1993; Davachi et al. 2003; Ranganath et al. 2004). In this study, contextual memory was tested by asking participants to judge in what condition the object was encoded. It is unclear whether the contextual memory can be enhanced after discriminative learning of similar objects. One possibility is that discriminating similar features would facilitate the binding process of various features of the object into a unified representation (Koutstaal et al. 1999), but that memory for the object's context would not be enhanced. The other possibility is that discriminating among similar features could also facilitate the binding of the features with their contexts, such as the position of the object, task related to the object, and other contextual information.

Second, we asked how participants encoded the similar and different pairs during discriminative learning and whether the encoding patterns for different conditions were associated with subsequent memory performance. To address these issues, we monitored eye movements in Experiment 2 when participants encoded similar and different pairs of objects. The eye-tracking technique has been used to reveal how memory is affected by previous experience and different conditions (e.g., Christianson et al. 1991; Ryan et al. 2000, 2007; Sharot et al. 2008; Hannula et al. 2012. For review, see Hannula et al. 2010). For example, in a study by Hannula et al. (2012), participants encoded faces and were subsequently tested using three-face displays. Half of the displays con- tained a studied face and similar faces; the other half displays contained three faces that were similar to the studied face. The results showed that the correctly identified faces attracted more viewing time than the faces mistakenly identified as studied. Advantages of this technique are that eye movements can be recorded over time and that the behavioral performance can be linked to parameters such as eye fixation location and time. These parameters are associated with attentional orienting and engagement when participants process online information (Rayner 1998, 2009; Henderson 2003). In Experiment 2, participants were asked to focus on one picture when the two pictures were presented together, then they were tested by the 2AFC task and contextual memory tasks. The parameters of the eye movements were analyzed to determine whether they were associated with subsequent memory performance.

We hypothesized that elaborative processing is more involved in the "similar" and "same" conditions than the "different" condition during discriminative learning, which leads to more distinct representations and higher item memory performance. In addition, compared to the "different" and "same" conditions, the participants are more likely to allocate attentional resources to compare the two similar objects during the "similar" condition (Koutstaal et al. 1999; Carvalho and Goldstone 2014) and establish relations between the objects and their contexts, which may lead to higher contextual memory performance. The eye movements during the discriminative learning would predict the subsequent item and contextual memory performance by allocating more fixations and fixation time on the lure pictures.

\section{Results}

\section{Experiment 1}

During the encoding phase, the participants judged whether the pairs of objects were in the same, similar or different condition. They performed the encoding task with high accuracy $(0.96 \pm$ $0.02)$. The accuracy was marginally different among the conditions $\left(F_{(2,44)}=5.28, P=0.09, \eta^{2}=0.21\right)$. The pairs in the "different" condition $(0.98 \pm 0.02)$ were judged more accurately than the other two conditions $(0.95 \pm 0.04$ and $0.95 \pm 0.03)\left(P^{\prime} s<0.05\right)$. There was no significant difference between the "same" and "similar" conditions $(P=0.72)$.

During the test phase, the participants were asked to perform the 2AFC task, followed by the contextual judgment at three retention intervals. Repeated-measures ANOVAs with encoding condition and retention interval as within-subject factors were performed. For the 2AFC, there was a significant effect of encoding condition $\left(F_{(2,44)}=31.44, P<0.001, \eta^{2}=0.59\right)$. Further analysis showed that memory performance was the highest for the "same" condition, followed by the "similar" condition, while it was the lowest for the "different" condition $\left(P^{\prime} \mathrm{s}<0.01\right)$. In addition, memory accuracy decreased over time $\left(F_{(2,44)}=16.94, P<\right.$ $\left.0.001, \eta^{2}=0.44\right)$. The interaction between encoding condition and retention interval was not significant $\left(F_{(4,88)}=1.02, P=0.40\right.$, $\eta^{2}=0.04$ ), although the difference between the "same" and "similar" condition trended toward being smaller over time (Fig. 1A). The results suggested that after discriminative learning of the "same" and "similar" pairs, the participants manifest better discrimination of detailed information of the objects than after learning of the "different" pairs, and this effect persisted for $1 \mathrm{wk}$. The accuracies in different conditions were significantly higher than a chance level $(0.50)\left(P^{\prime} s<0.05\right)$, except in the "different" condition at $1 \mathrm{wk}(P=0.54)$.

With regard to contextual memory, the results showed that there was a significant effect of encoding condition $\left(F_{(2,44)}=\right.$ $\left.13.26, P<0.001, \eta^{2}=0.38\right)$. Further analysis showed that contextual 

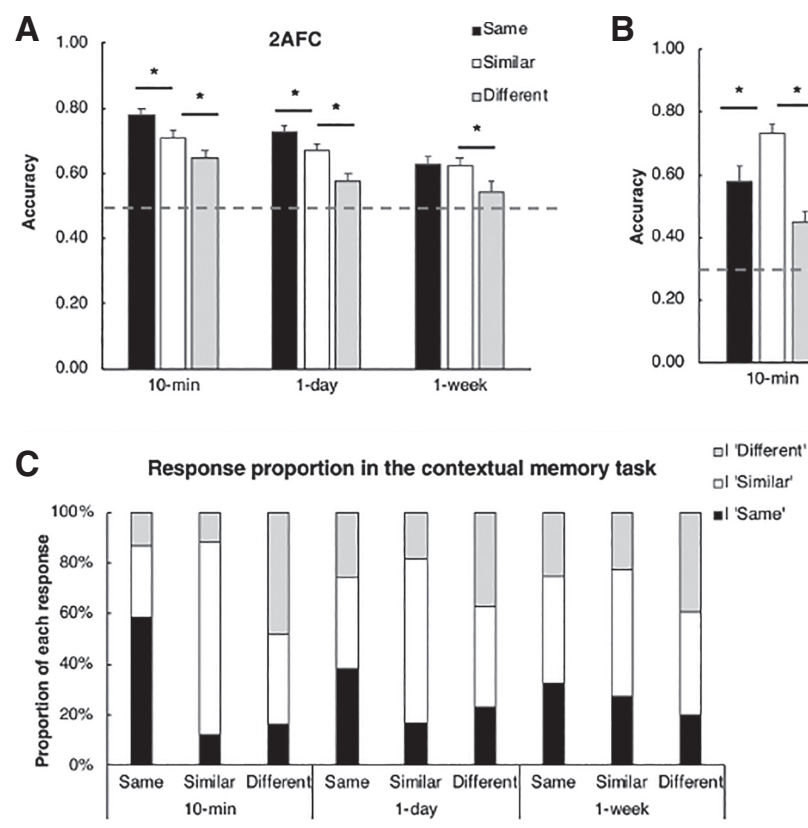

Figure 1. Behavioral results in Experiment 1. Memory accuracy was higher for the "similar" than the "different" condition in the 2AFC task $(A)$ and the contextual memory task $(B)$. The error bars represent the standard errors of the means. The dotted lines represent the chance level in each task. The lines with * represent significant differences between the "similar" and "different" conditions or between the "similar" and "same" conditions $(P<0.05)$. The response proportion in the contextual memory task is also illustrated $(C)$.

memory was the best for the "similar" condition $\left(P^{\prime} \mathrm{s}<0.001\right)$, and comparable for the "same" and "different" conditions $(P=0.61)$ (Fig. 1B). In addition, memory accuracy decreased significantly over time $\left(F_{(2,44)}=23.36, P<0.001, \eta^{2}=0.52\right)$. The interaction between condition and retention interval was not significant $\left(F_{(4,88)}=2.52, P=0.08, \eta^{2}=0.10\right)$. The results suggested that discriminative learning of similar objects enhances the contextual memory and persists for $1 \mathrm{wk}$.

The contextual memory was significantly higher than a chance level (0.33) for the "similar" conditions at each retention interval $(P$ 's $<0.05)$, but only significantly higher than chance level for the "same" and "different" conditions at 10 -min interval $\left(P^{\prime} s<0.05\right)$. The proportion of the response type for each condition was also illustrated in Figure 1C. Note that the same "similar" condition at $1 \mathrm{wk}$ was higher than the chance level $(0.42 \pm 0.18, P=0.03)$, suggesting that for the object in the "same" condition, the participants are more likely to incorrectly judge it from the "similar" condition at 1-wk interval.

In sum, the main results of Experiment 1 showed that compared to the "different" condition, the discriminative learning of the "same" and "similar" conditions enhanced subsequent item memory. In addition, the "similar" condition enhanced contextual memory compared to the other two conditions. Even at 1-wk interval, the detailed memory
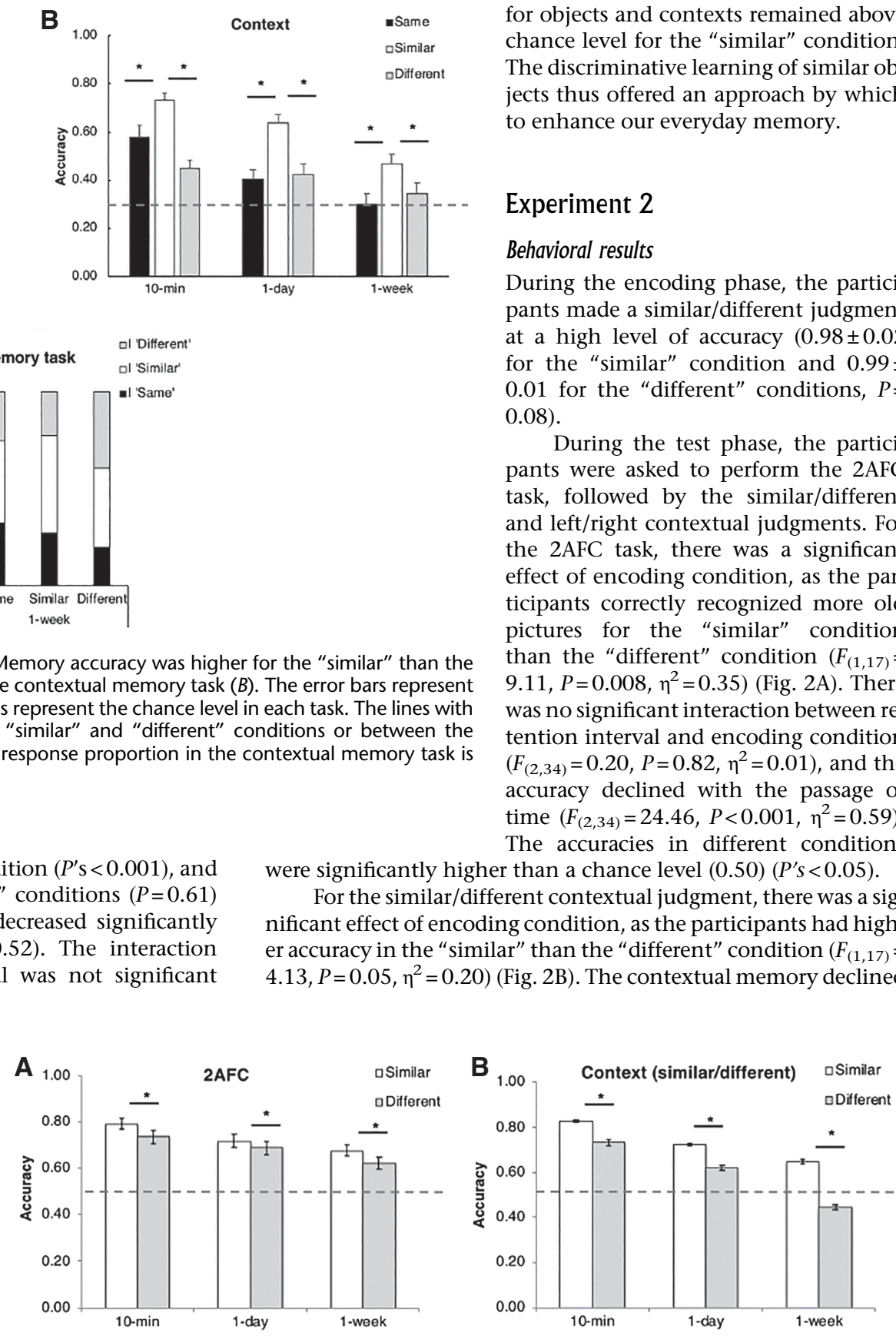

for objects and contexts remained above chance level for the "similar" condition. The discriminative learning of similar objects thus offered an approach by which to enhance our everyday memory.

\section{Experiment 2}

\section{Behavioral results}

During the encoding phase, the participants made a similar/different judgment at a high level of accuracy $(0.98 \pm 0.02$ for the "similar" condition and $0.99 \pm$ 0.01 for the "different" conditions, $P=$ 0.08).

During the test phase, the participants were asked to perform the $2 \mathrm{AFC}$ task, followed by the similar/different and left/right contextual judgments. For the 2AFC task, there was a significant effect of encoding condition, as the participants correctly recognized more old pictures for the "similar" condition than the "different" condition $\left(F_{(1,17)}=\right.$ 9.11, $P=0.008, \eta^{2}=0.35$ ) (Fig. 2A). There was no significant interaction between retention interval and encoding condition $\left(F_{(2,34)}=0.20, P=0.82, \eta^{2}=0.01\right)$, and the accuracy declined with the passage of time $\left(F_{(2,34)}=24.46, P<0.001, \eta^{2}=0.59\right)$. The accuracies in different conditions were significantly higher than a chance level $(0.50)\left(P^{\prime} s<0.05\right)$.

For the similar/different contextual judgment, there was a significant effect of encoding condition, as the participants had higher accuracy in the "similar" than the "different" condition $\left(F_{(1,17)}=\right.$ $4.13, P=0.05, \eta^{2}=0.20$ ) (Fig. $2 \mathrm{~B}$ ). The contextual memory declined 
with the passage of time $\left(F_{(2,34)}=31.40\right.$, $\left.P<0.001, \eta^{2}=0.65\right)$. There was no significant interaction between retention interval and encoding condition $\left(F_{(2,34)}=\right.$ 2.39, $P=0.13, \eta^{2}=0.12$ ). The accuracies in different conditions were significantly higher than a chance level $(0.50)\left(P^{\prime} s<\right.$ $0.05)$ except in the "different" condition at 1 -wk interval $(P=0.23)$.

For the left/right position judgment, there was a significant interaction between retention interval and encoding condition $\left(F_{(2,34)}=5.46, P=0.01, \eta^{2}=\right.$ 0.24 ), insofar as the memory performance was higher after the "similar" condition than the "different" condition at 10-min and 1-wk intervals $\left(P^{\prime} \mathrm{s}<0.05\right)$ (Fig. $\left.2 \mathrm{C}\right)$. The memory declined with the passage of time $\left(F_{(2,34)}=39.13, \quad P<0.001, \quad \eta^{2}=\right.$ $0.70)$. The accuracies in different conditions were significantly higher than a chance level $(0.50)\left(P^{\prime} s<0.05\right)$, except in the "different" condition at 1-wk interval $(P=0.75)$. The results suggested that, similar to the case of the similar/different judgment, comparing between the similar objects during encoding enhances subsequent memory for position contexts.

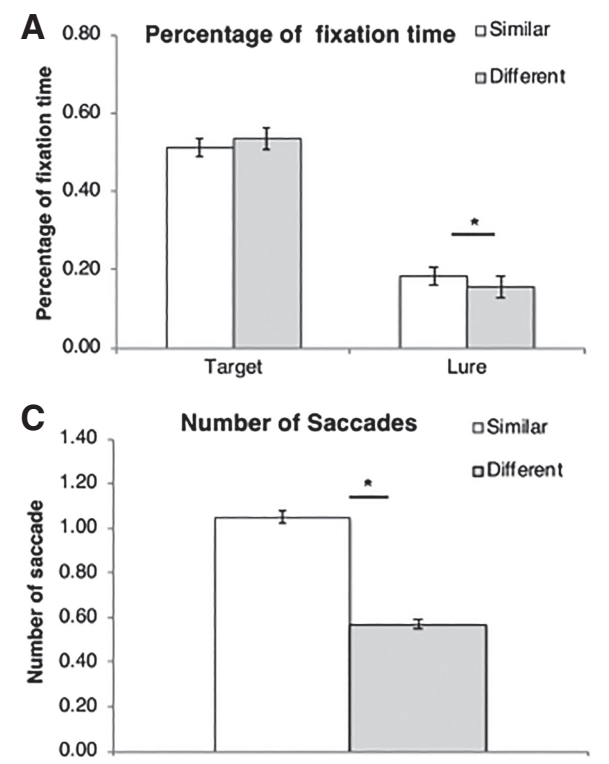

B Percentage of fixation number aSimilar

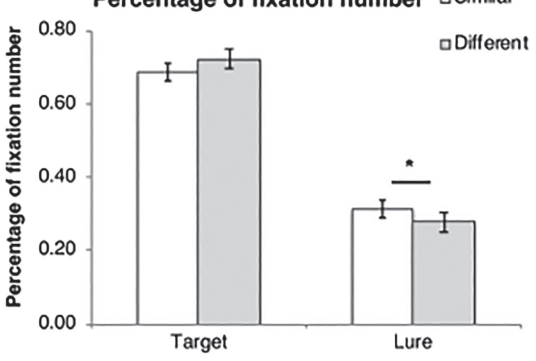

Figure 3. Eye-movement results in Experiment 2. For the target pictures, the eye parameters were comparable for the "similar" and "different" conditions, but for the lure pictures, the eye parameters were higher for the "similar" than for the "different" conditions. This pattern was manifested for the percentage of fixation time $(A)$ and the percentage of number of fixations $(B)$. There was significantly more number of saccades in the "similar" than the "different" condition $(C)$. The error bars represent the standard errors of the means. The lines with * represent significant differences between the "similar" and the "different" condition $(P<0.05)$.

\section{Eye movement during encoding}

An ANOVA of encoding condition (similar, different) and picture type (target, lure) was performed for the percentage of fixation time, percentage of the number of fixations, number of gaze fixations, and total fixation time. The four parameters showed similar results.

For the percentage of fixation time, there was a significant effect of picture type $\left(F_{(1,16)}=58.33, P<0.001, \eta^{2}=0.79\right)$. This was mainly because the participants were asked to remember the target pictures during encoding. The difference between the two encoding conditions was not significant $\left(F_{(1,16)}=0.091, P=0.78, \eta^{2}=\right.$ $0.01)$, but importantly, there was a significant interaction between condition and picture type $\left(F_{(1,16)}=7.41, P=0.02, \eta^{2}=0.32\right)$. Further analysis showed that for the target pictures, the percentage of fixation time was comparable for the "similar" and "different" conditions $(P=0.07)$, but for the lure pictures, it was higher for the "similar" than for the "different" condition $(P=0.02)$ (Fig. $3 \mathrm{~A})$. Similarly, for the percentage of number of fixations, the effect of picture type $\left(F_{(1,16)}=48.12, P<0.001, \eta^{2}=0.75\right)$ and the interaction between encoding condition and picture type were also significant $\left(F_{(1,16)}=5.33, P=0.04, \eta^{2}=0.25\right)$. Further analysis showed that for the target pictures, the percentage of number of fixations was comparable for the "similar" and "different" conditions $(P=$ $0.47)$, but for the lure pictures, it was higher for the "similar" than for the "different" condition $(P=0.01)$ (Fig. 3B). The findings were the same for the number of fixations and fixation time. These results suggested that the participants focus their attention more on the lure pictures in the "similar" condition than in the "different" condition.

We also noted that the participants allocated their fixation time outside of the targets and lures $(1455.26 \pm 758.52 \mathrm{msec}$ for the "similar" condition and $1460.78 \pm 889.54 \mathrm{msec}$ for the "different" condition, $P=0.97)$. It is possible that because the participants had seen the two pictures for $1.5 \mathrm{sec}$ previously, they may have tried to remember the target by not looking at either of the pictures.
For the number of saccades, the results showed that there were significantly more saccades in the "similar" condition than in the "different" condition $\left(t_{(16)}=6.93, P<0.001\right)$ (Fig. 3C). It suggested that compared to the "different" condition, there are more elaborative processing in the "similar" condition to discriminate the target from the lure pictures.

\section{Eye movement and $\mathrm{Dm}$ effects}

To explore whether the eye-movements predicted the subsequent memory performance (i.e., difference in memory, Dm), we defined the Dm effect as the proportion of subsequently remembered items minus the proportion of subsequently forgotten items. We first examined whether the Dm effect was present or absent. Then, we conducted a repeated-measures ANOVA with the factors of encoding condition (similar, different), picture type (target, lure), and retention interval for the proportion of fixation time.

For the 2AFC task, the Dm scores were at chance levels in all conditions $\left(t\right.$ 's $\left.<1, P^{\prime} s>0.30\right)$, suggesting the absence of a subsequent memory effect. There were no significant effects for the factors nor their interaction $\left(F^{\prime} \mathrm{s}<1, P>0.10\right)$ (Fig. 4A). For the similar/ different judgment task, the Dm score for the lure pictures in the "similar" condition was significantly above the chance level $\left(t_{(16)}=2.11, P=0.05\right)$, but this was not true for the other conditions $\left(P^{\prime} s>0.80\right)$. There was a significant interaction between encoding condition and target type $\left(F_{(1,16)}=11.71, P=0.003, \eta^{2}=0.42\right)$, as the proportion of the fixation time on the lure pictures was higher for the "similar" than the "different" condition $(P<0.02)$ (Fig. 4B), but this pattern did not occur for the target pictures. It suggested that the more fixation time there is on the lure pictures, the more likely the task contexts will be remembered. For the left/ right judgment task, the Dm score was significantly above the chance level $\left(t_{(16)}=2.87, P=0.01\right)$ for the target pictures in the "different" condition (Fig. 4C), but this was not the case for the other 

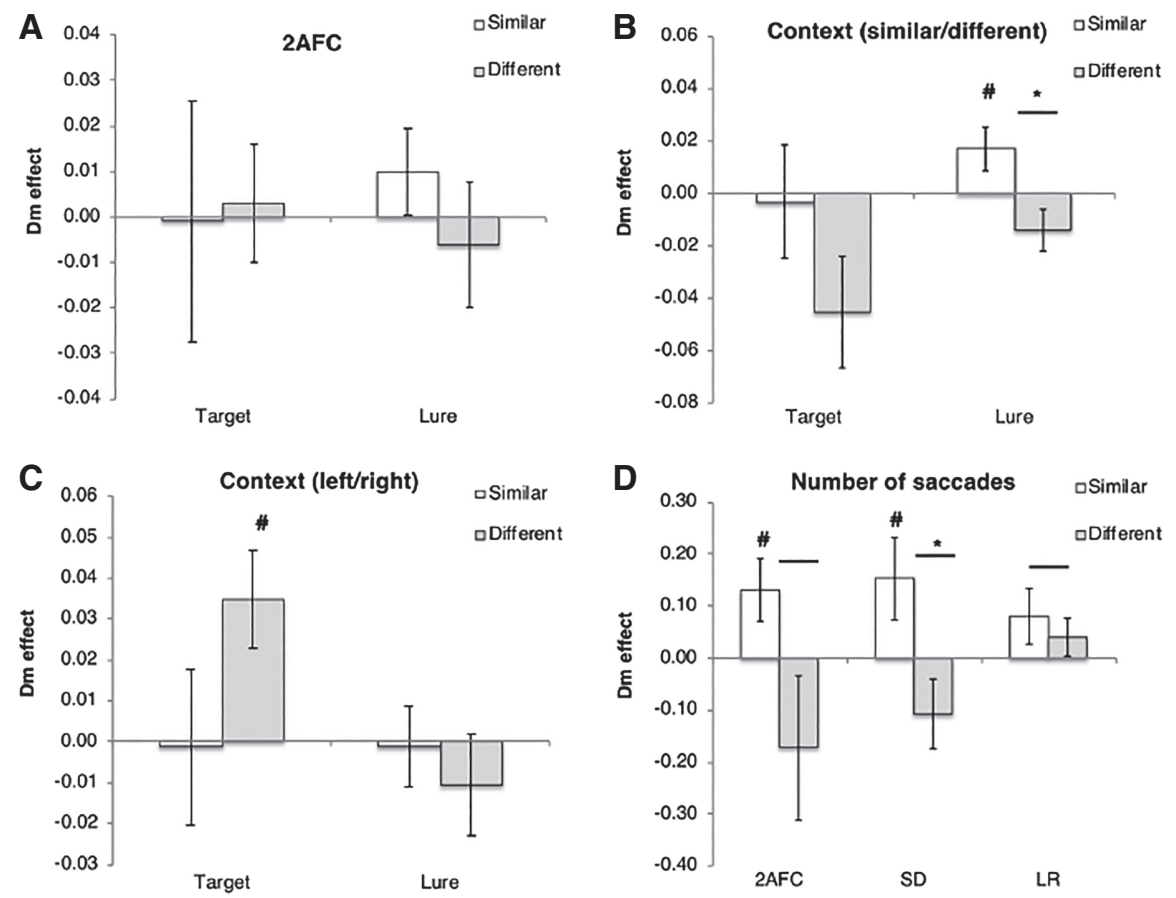

Figure 4. Results of the Dm effects in Experiment 2. The Dm effects for the percentage of fixation time are shown in A-C. The Dm effect was higher than chance level for the lure pictures in the "similar" condition, and it was higher under the "similar" condition than the "different" condition in the similar/different contextual memory task $(B)$. Similar patterns were manifested when the number of saccades $(D)$ was analyzed in the 2AFC task, similar/different judgment task (i.e., SD) and the left/right judgment task (i.e., LR). The error bars represent the standard errors of the means. The lines with * represent significant differences between the "similar" and "different" conditions $(P<0.05)$. The lines without * represent marginally significant differences between the "similar" and "different" condition. The "\#" represents a significant difference from chance level $(P<0.05)$.

conditions $\left(P^{\prime} S>0.20\right)$. There were no significant effects for the factors nor their interaction $\left(P^{\prime} \mathrm{s}>0.15\right)$.

The number of saccades was also analyzed by ANOVA with encoding condition and retention interval as factors for the Dm effect (Fig. 4D). For the 2AFC task, the Dm score was significantly greater than the chance level for the "similar" condition $\left(t_{(16)}=2.13, P=\right.$ $0.05)$, but not for the "different" condition $(P=0.22)$. There was a marginally significant effect of condition $\left(F_{(1,16)}=3.44, P=0.08\right.$, $\eta^{2}=0.18$ ), showing that more saccades in the "similar" than the "different" condition predicted the Dm effect. Although the difference was marginally significant, the medium effect size indicated the result was reliable. The interaction between condition and retention interval was not significant $\left(F_{(2,32)}=0.55, P=0.58, \eta^{2}=\right.$ $0.03)$. For the similar/different judgment task, the Dm score was significantly greater than the chance level for the "similar" condition $\left(t_{(16)}=2.13, P=0.05\right)$, but not for the "different" condition $(P=0.12)$. There was a significant effect of condition $\left(F_{(1,16)}=\right.$ $7.01, P=0.02, \eta^{2}=0.31$ ), showing that more saccades in the "similar" (versus "different") condition predicted the Dm effect (Fig. 4D). For the left/right judgment task, the Dm score was marginally significant above the chance level for the "similar" condition $\left(t_{(16)}=1.88, P=0.08\right)$, but not for the "different" condition $(P=0.19)$. There was a marginally significant effect of condition $\left(F_{(1,16)}=2.51, P=0.08, \eta^{2}=0.14\right)$, showing that more saccades in the "similar" (versus "different") condition predicted the Dm effect. The interactions between encoding condition and retention interval for the two contextual tasks were not significant $\left(F^{\prime} s<1\right.$, $\left.P^{\prime} s>0.40\right)$. The results suggested that the number of saccades predicts whether the target picture is remembered during later $2 \mathrm{AFC}$ and source memory tasks.
In sum, the results of Experiment 2 showed that both item and contextual memory performance was higher after the discriminative learning of the similar (versus different) pictures. The participants allocated more eye fixations and fixation time to the lure pictures and made more saccades between the two pictures in the "similar" (versus "different") condition. The number of saccades between the two pictures predicted that the target pictures and the contexts would be remembered in the subsequent tests. These eye movements enabled the participants to compare the two pictures during encoding and enhanced subsequent memory.

\section{Discussion}

In this study, by the discriminative learning of similar objects, the object and its context were better remembered and retained over time. In addition, the participants were more likely to move their eyes to the lure pictures during the discrimination of similar objects, and the number of saccades predicted how well the targets were remembered in both the 2AFC and contextual tasks. The results clarified how discriminative learning modulated the encoding process for the memory enhancement in both item and contextual dimensions.

\section{Enhanced memory for object details}

One of the novel findings of the study was that discriminative learning of the similar or same (versus different) pairs enhanced the 2AFC task performance. The results suggested that discriminative learning of the same and similar objects elicits more elaborative and distinctive processing. Higher levels of elaboration during encoding is thought to produce detailed and distinctive representations, and these enriched descriptions help distinguish elaborated items from other candidate items at the time of retrieval (Lockhart and Craik 1990). If the objects belong to the same concept, the attentions will be focused on their perceptual difference (Carvalho and Goldstone 2014) and the encoding process will be more distinctive. In contrast, if the objects belong to different concepts, general or conceptual knowledge of the objects is obtained, with less detailed information for each picture being processed. During retrieval, two similar objects were presented. One was seen during encoding, and the other was new. The details of the learned object could facilitate the judgment of which object was seen previously. Studies also have shown that blocked presentations (Carvalho and Goldstone 2014) and instructions on encoding details (Koutstaal et al. 1999) facilitate subsequent memory performance. The results of the present study further showed that the details of the objects could be better processed and remembered in the "same" and "similar" conditions compared to the "different" condition.

The results of the eye-tracking data confirmed that during encoding, presenting two similar objects facilitated attentional allocation and, hence elaborative processing. Although the participants fixated on the targeted object as instructed, they were more likely to move their eyes to the lure object to make a comparison in 
the "similar" condition compared to the "different" condition. The attention allocation may enhance the elaborative processing of detailed information. The number of saccades between the target and the lure pictures also predicted how well the target was remembered in the later 2AFC test. Sharot et al. (2008) suggested that remembering is related to encoding a few distinct details of a picture rather than the picture as a whole. They found that fixations were fewer and more clustered during both the encoding and recognition of remembered pictures relative to known ones. In turn, during recognition, remembering may be trigged by an enhanced memory for the salient details of the pictures.

The results also showed that enhanced memory in the "same" and "similar" (versus "different") conditions persisted for $1 \mathrm{wk}$. It suggested that, via elaborative encoding, a distinctive representation may be quickly formed and consolidated that is resistant to memory decay over time (Sadeh et al. 2014). It is widely assumed that our memory loses details with the passage of time. However, some studies have suggested that long-term memory is capable of storing a massive number of objects with details from the images (Brady et al. 2008; Huebner and Gegenfurtner 2012; Andermane and Bowers 2015). Our results further suggested that by discriminating perceptually same or similar pairs, detailed information about the objects is encoded and the memory enhancement persists over time.

Discriminative learning of similar contexts has been used in animal studies. In such studies, animals could remember the fear contexts for a long time, even without the involvement of the hippocampus (McHugh et al. 2007; Sahay et al. 2011; Nibori et al. 2012; Czerniawski and Guzowski 2014). We applied the discriminative learning paradigm to a human population and found significant improvement in memory performance. The discriminative learning of similar objects is an efficient way to retain detailed memories for individual objects in both animal and human studies.

\section{Enhanced memory for object contexts}

Another novel finding of the study was that the contextual memory was enhanced after discriminative learning of the "similar" pairs (versus "same" and "different" pairs). The results suggested that via discriminative learning, the participants not only form a distinctive representation of an object but also establish a relational representation between the object and its contexts.

Contextual memory is different from item memory in both cognitive and neural mechanisms. For example, in the similar/ different judgment task, the participants had to associate the task information with the item when they recognized that the item was seen during encoding. Thus, the recollection process contributes more sizably to contextual memory, and the hippocampus is more involved in this process compared to item memory (Cohen and Eichenbaum 1993; Eichenbaum et al. 1994; Davachi et al. 2003; Ranganath et al. 2004; Eichenbaum et al. 2007). In this study, the contextual information we tested included the condition during encoding and the position in which the picture was placed during encoding. Note that the contextual information was not intrinsic to the item itself; thus, only the distinctive representation of the object could not support the contextual memory. We found enhanced contextual memory for the "similar" condition in both experiments. This suggested that by discriminating between similar pictures, the contextual information is covertly processed in addition to the details of an object.

The results of the eye-movements showed that the number of saccades during encoding predicted subsequent contextual memory for the "similar" condition. This finding indicated that saccades are important for forming an association between an object and its contextual information (e.g., task performed, target position). Eye movements, including saccades, represent an overt behavioral manifestation of the allocation of attention (Henderson 2003), and shifts in attention may support the integration of information across saccades (Melcher and Colby 2008; Kowler 2011). In the present study, although the target object was designated, the participants allocated more fixations and time to the lure object, which led to a greater number of saccades between the target and lure objects. Saccades have been shown to be associated with successful encoding (Irwin 1991; Hollingworth et al. 2008) and memory processes (Henderson and Hollingworth 2003; Hannula et al. 2010). For example, when subjects learned an array of objects, they spontaneously looked backward in their scanning paths to view recently seen objects again, and this revisit was associated with hippocampal activity and higher subsequent memory performance (Voss et al. 2011). The contextual information is therefore integrated with the object across the saccades (Melcher and Colby 2008; Kowler 2011), and this process is associated with subsequent contextual memory.

One interesting result was that we observed enhanced source memory for the "similar" condition but not for the "same" condition. This was different from the result obtained during the 2AFC task, in which memory performance was better for the "same" condition than for the "similar" condition. During the encoding of two identical images, the participants had to process every detail to ensure that the two objects were the same. However, simple repetition and processing details of single objects did not enhance contextual memory, as the contextual memory performance was comparable for the "same" and "different" conditions, and the performance was at chance level at 1-d and 1-wk intervals. It is possible that when the two similar objects are presented, there is more contextual information being processed to facilitate the associations between the objects and various contexts (Frankland et al. 1998), even if participants are not asked to intentionally process them. The eye-tracking analysis showed that when asked to remember a target object, the participants allocated more attention to it but still moved their eyes to make a comparison under the "similar" condition. Thus, during the contextual memory task, there is more associated information being reactivated to facilitate the contextual judgment.

Note that the enhanced source memory was maintained over the 1-wk interval, although the performance still declined. This is important because the contextual memory is usually forgotten very quickly (Sadeh et al. 2014; Moscovitch et al. 2016) if memory is not selectively enhanced. Different types of memory have distinctive forgetting rates (Hockley and Consoli 1999). Item memory is familiarity-based and is resistant to decay over time. Once the item memory is established, it can be retained for a long time. Contextual memory, on the other hand, is more likely to decay with the passage of time (Sadeh et al. 2014). The discriminative learning of similar objects enhanced both types of memory. Thus, by discriminating between "similar" objects, both item and contextual memory can be enhanced and retained for a long time.

\section{Discriminative learning and future directions}

To our knowledge, this is the first study that used the discriminative learning paradigm to explore its effects on item memory and contextual memory in humans. The mechanism of discriminative learning of similar objects was consistent with the level of processing (LOP) framework (Craik and Lockhart 1972; Lockhart and Craik 1990). Based on the LOP framework, discriminative learning induces elaborative encoding. Elaboration refers to the degree to which each type of processing is enriched during encoding (Lockhart and Craik, 1990). By discriminative encoding, a richly elaborated trace is more differentiated from other episodic records, and its distinctiveness supports more effective recollection of the 
stimulus (Lockhart and Craik 1990; Moscovitch and Craik 1976). In addition, elaborative traces are more integrated with contextual information to facilitate subsequent contextual memory.

The current study provided a useful attempt to find behavioral difference in the discriminative encoding of different types of stimuli. Discriminative learning of similar objects improved memory of the object details and contextual details. The results of eye movements provided further evidence to explains how discriminative encoding enhanced subsequent memory-namely, participants are more likely to distinguish a target from its distractor by moving their eyes to compare them, and to associate the target with its contextual information by more saccades. This differs from the typical LOP manipulation, in which the same stimuli are encoded by different levels of tasks. In the discriminative learning paradigm, different types of stimuli are encoded by the same task. This is similar to the self-referential effect (Lockhart and Craik 1990), which is a promising way to index the processing depth of behavior.

There are some limitations to be addressed by future investigations. First, we did not record RTs or self-confidence during encoding in either experiment. This limits our explanation for whether there are differences between conditions or whether the differences are associated with subsequent memory performance. During retrieval, we only included similar objects as lures. Not including lures from different categories may raise the possibility that enhanced memory is related to the encoding-retrieval congruency. Second, during eye-tracking in Experiment 2, approximately $30 \%$ of the total duration was located outside of the AOIs, partly because the participants had already seen the pictures for $1.5 \mathrm{sec}$ before. The one-presentation is necessary to verify that the eye-tracking results were not confounded by the previous presentation. Third, we did not find a significant interaction between encoding condition and retention interval in either experiment for the 2AFC. One possibility is that because the familiarity process mainly contributes to the 2AFC test (Holdstock et al. 2002; Norman and O'Reilly 2003; Migo et al. 2009), the 2AFC test may not sensitive to memory forgetting (Sadeh et al. 2014). Further studies could use other memory tests (e.g., recognition, recall) that are sensitive to the recollection process to address this issue (Huebner and Gegenfurtner 2012; Andermane and Bowers 2015).

\section{Conclusions}

By distinguishing between the simultaneously presented objects, participants improved their memory performance in the 2AFC and contextual memory tasks in the "similar" condition more than in the "different" condition. This pattern occurred across different retention intervals. The number of saccades predicted how well the targets were remembered in both the 2AFC and contextual tasks. These results have significant implications. Because remembering detailed information is important and subject to interference and aging, this paradigm provides a promising way to improve the memory for detailed information over time, which could be applied to the general population and those who have memory deficits.

\section{Materials and Methods \\ Experiment 1}

\section{Participants}

Twenty-three healthy, right-handed participants (10 males) with a mean age of $21.75 \pm 2.02 \mathrm{yr}$ were recruited in the study. All of the participants were native Chinese speakers, and they all provided written informed consent in accordance with the procedures and protocols approved by the Review Board of Peking University.

\section{Material}

Two within-subject factors were included in the study: encoding (same, similar, different) and retention interval (10 min, $1 \mathrm{~d}$, and $1 \mathrm{wk})$.

We selected 720 objects (240 triplets) from Hemera Photo Clipart and from the Internet. Each triplet included three similar but slightly different color pictures with the same basic concept/ name (e.g., dog, tomato). The three pictures used in the experiment had clear and easy-to-be-extracted concepts, and they differed in dimensions such as orientation, color, and the number. They were of equal size $(640 \times 480$ pixels $)$, with white backgrounds.

The 720 pictures were selected from an original pool of 1344 pictures (448 triplets) based on the results of their naming performance, ratings of familiarity, and similarity within the triplets. A total of 23 subjects ( 12 males, mean age of $22.83 \pm 2.67 \mathrm{yr}$ ) who did not participate in the experiments were recruited for the picture naming and rating tasks. In the first phase, the participants performed the naming task for each picture, then they rated the familiarity (one for most familiar, five for most unfamiliar) of the same picture. In the second phase, the participants rated the similarity within the triplets in a different order. In this phase, two pictures of the same concept were presented on the screen (i.e., the pictures in each triplet had three pairs). The participants rated how similar the two pictures were on a five-point Likert scale. Three ratings for every two pictures of a triplet were acquired and averaged as one score for the similarity within the triplet of each concept. The orders of the pictures were pseudorandomized in the two phases so that no consecutive trials were of the same concept. The selection was triplet-wise, with the three pictures of a triplet either all selected or all excluded. The naming accuracy was $0.91 \pm 0.12$, familiarity was $1.81 \pm 0.33$, and the similarity score was $2.93 \pm 0.51$.

All selected triplets were first randomly assigned to four groups (Groups A, B, C, and D) (60 triplets per group), with one group used for the "same" condition, one for the "similar" condition and the other two for the "different" condition. For the "different" condition, two pictures were selected from the two groups. Then, each group was further assigned to three different sets (S1, 2, and 3) for three retention intervals (20 triplets per set). The three pictures within a triplet were separated into three subsets within each set to be used as the old and lure pictures in the test (20 pictures per subset). For each set, the pairs in the test were organized as follows: for the "same" condition (A1-A1), the pictures in one subset were used during encoding to form the same pairs. During testing, the picture was paired with a picture from a similar picture from one the other two subsets (A2 or A3). For the "similar" condition (B1-B2), the pictures from two subsets were paired within the same concept during encoding. The pictures in the third subset (B3) were used to form the test pairs with one of the old pictures (B1-B3). For the "different" condition (C1-D1), one subset in each of the two groups was randomly paired during encoding. One of the other subsets (C2 or C3) from the same group was used to form test pictures with one of the old pictures (e.g., C1). The material in groups, sets, and subsets was counterbalanced across participants so that each picture had the same chance to be used for each condition. There were no significant differences in naming performance, familiarity, or similarity within triplets across groups, sets or subsets $\left(P^{\prime} \mathrm{s}>0.60\right)$.

\section{Procedure}

The participants learned the 180 pairs on the same day, and then performed the $2 \mathrm{AFC}$ and source memory tasks at three retention intervals (60 pairs per retention interval, 20 pairs per condition) (Fig. 5A). During the study phase, for each trial, the picture pair (same, similar and different) was presented in the center of the screen for $3 \mathrm{sec}$, while the participants were asked to judge whether the two pictures were the same, similar or different (Fig. 5B). The order of the three buttons was counterbalanced across the participants. All pairs were pseudorandomly presented during the encoding phase so that no more than three stimuli that were in the same condition were presented consecutively. For the "similar" and the "different" conditions, the position of the target/old picture (that 
A

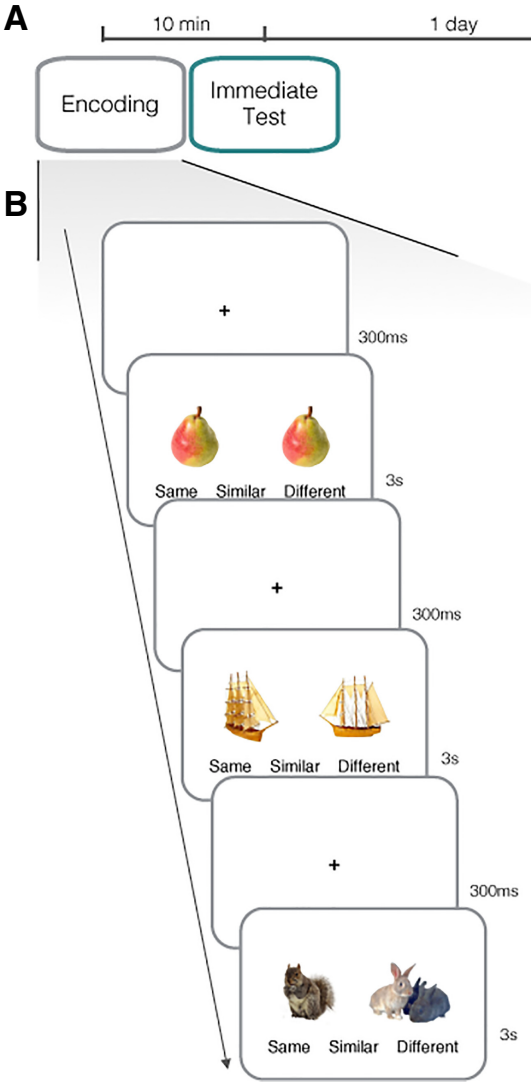

Figure 5. Procedure of Experiment 1 . The procedure included one study phase and three test phases $(A)$. During the study phase, participants were asked to judge whether the two pictures were the same, similar or different $(B)$. During each of the test phases, the participants finished a 2AFC task and a contextual memory task (C).

appeared in the test) was randomly presented on the left or right of the pair and counterbalanced across the participants.

During the test phase, the presented pair was composed of an old picture and a similar/lure picture. Each pair was presented in the center of the screen for $3 \mathrm{sec}$, and the participants finished a 2AFC task during which they were asked to choose the picture learned during the encoding phase as quickly and accurately as possible, followed by a confidence rating of 1-5 (Fig. 5C). Then, they were asked to judge whether the old picture appeared in the "same," a "similar," or a "different" condition during encoding, followed by a $1-5$ confidence rating. Half of the old pictures were located on the left, and the other half were on the right. The positions of the old pictures were counterbalanced across the participants. All pairs were pseudorandomly presented at each retention interval so that no more than three pairs in the same condition were presented consecutively.

Before each test phase, to avoid rehearsing the stimuli learned in the study phase, the participants were asked to count backward by seven continuously from 1000 for $5 \mathrm{~min}$. The participants had separate opportunities to practice study and test trials before the formal phase.

\section{Data analysis}

The accuracies of the 2AFC task and the contextual memory task for all trials were calculated and analyzed using a repeated-measures ANOVA with the retention interval and encoding conditions as within-subject factors. The accuracies for the high-confidence (HC) responses (i.e., rating from 4-5) were also analyzed for the two tasks. As the $\mathrm{HC}$ results were similar to those including all trials, they were not reported. The accuracy of contextual memory re- ferred to the number of correct trials in the contextual judgment out of the number of correct trials in the 2AFC, and out of the total number of trials in each condition. As the results were similar, only the former was reported. The forgetting rate was estimated by the interaction between the retention interval and the condition (Slamecka and McElree 1983; Gardiner and Java 1991; Hockley and Consoli 1999). Partial Eta Squared $\left(\eta^{2}\right)$ was calculated to estimate the effect size of each analysis. Post hoc pairwise comparisons were Bonferroni corrected $(P<$ 0.05, two-tailed).

\section{Experiment 2}

\section{Participants}

Eighteen participants (10 males) with a mean age of $21.89 \pm 2.99 \mathrm{yr}$ were recruited in the study. All of the participants were native Chinese speakers, and they all gave written informed consent in accordance with the procedures and protocols approved by the Review Board of Peking University.

\section{Materials}

Two within-subject factors were included in the study: encoding (similar, different) and retention interval (10 min, $1 \mathrm{~d}, 1 \mathrm{wk}$ ).

The materials were the same as those in Experiment 1. Because the 2AFC memory performance was better for both the "same" and "similar" conditions than for the "different" condition, and the contextual memory was better for the "similar" than the "same" condition in Experiment 1, we did not include the "same" condition in Experiment 2. This also ensured that there was a sufficient number of trials to be used for the analysis of the subsequent memory effect. All triplets were divided into two groups (120 triplets per group), with one used for the "similar" condition and the other for the "different" condition. Then, each group was divided into three sets to be used for the retention intervals (40 triplets per interval). During encoding, for the "similar" condition, two pictures from the 20 triplets were presented; one of them been randomly assigned as the target and the other as the lure picture. Thus, half of the triplets within the "similar" condition were not used. The triplets were counterbalanced across the participants. For the "different" conditions, two pictures from different triplets were paired: one of them was randomly assigned as the target and the other as the lure picture. During the test phase, the target pictures were paired with the third picture in the triplets for the "similar" condition, and with one of the other two unused pictures within the triplets for the "different" condition. The material in groups, sets and subsets was counterbalanced across participants so that each picture had the same chance to be used for each condition. There were no significant differences in naming performance, familiarity or similarity within triplets across groups, sets or subsets $\left(P^{\prime} \mathrm{s}>0.10\right)$.

\section{Procedure}

During encoding, an eye-tracking technique was used. For each trial, the picture pair (similar or different) was presented in the center of the screen for $1.5 \mathrm{sec}$, while the participants were asked to judge whether the two pictures were similar or different (Fig. 6A). Then, the two pictures were presented again for $4 \mathrm{sec}$, and one of them was randomly labeled as the target. The participants were asked 
A

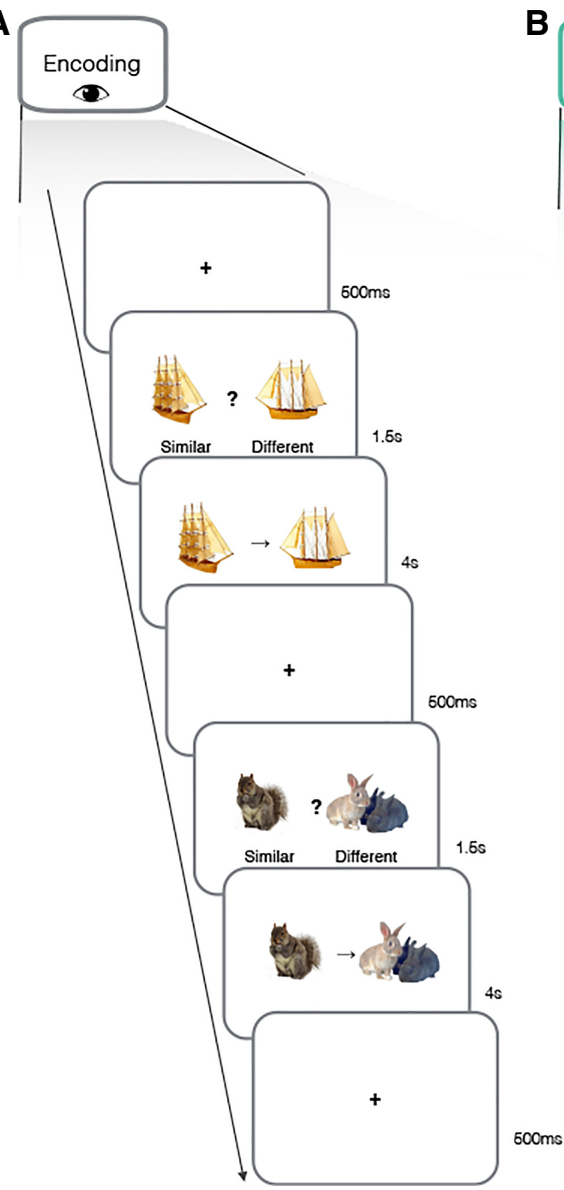

Figure 6. Procedure of Experiment 2. During the study phase, participants were asked to judge whether the two pictures were similar or different, then they were asked to remember the target picture $(A)$. During the test phase, the participants finished the 2AFC task and the contextual memory tasks $(B)$.

to remember the target picture. During testing, the old and similar pictures were presented together, and the participants were asked to make a forced choice for the old picture. After that, two contextual memory tasks were performed: the similar/different judgment and the left/right judgment. Each task was followed by the confidence rating. To examine whether the advantage of contextual memory was specific to the task manipulation, we asked the participants to perform an additional task, i.e., a left/right position judgment of the old object. During the left/right judgment, the subjects judged which side of the screen the target image was on during encoding (Fig. 6B). The order of the two source tasks was counterbalanced across the participants. In each memory test, 40 targets were tested, 20 for each condition. The participants learned a total of 240 pictures during encoding, 120 for the "similar" condition and 120 for the "different" condition. Within each condition, the target was presented on the left of the screen for half of the trials and on the right for the other half. The order of the presentation was pseudorandomized so that no more than three consecutive trials were of the same condition or of the same position of target.

\section{Eye-tracking apparatus}

The eye-movement was recorded during the encoding phase. Stimuli were presented on a 17 -inch ViewSonic monitor with a resolution of $1024 \times 768$ pixels. The participants sat on a chair and placed their head on a chin rest so that their heads were $60 \mathrm{~cm}$ away from the viewing screen. Eye movements were monitored using an SR Research Eyelink 1000 connected to a computer with a sampling rate of $1000 \mathrm{~Hz}$. The participants viewed the pictures binocularly, but only their left eye movements were monitored. The eye-tracker was calibrated prior to picture presentation using a ninepoint matrix.

\section{Data analysis}

The behavioral data analysis was the same as that in Experiment 1 . The accuracies for the 2AFC and contextual memory tasks for all trials were analyzed by ANOVA, with retention interval and encoding condition as two within-subject factors $(P<0.05$, two-tailed) and reported in the paper. In analyzing the eyemovement data, the areas of interest (AOIs) were defined as the positions where the two pictures (i.e., target picture and lure picture) were presented, and the size was the same as the pictures. All trials were included in the analysis. The eyetracking parameters included the number of gaze fixations, fixation time, percentage of the number of fixations and fixation time (out of the total number of fixations or the total fixation time, respectively) in the two AOIs (Calvo and Lang 2004; Chua et al. 2005; Nummenmaa et al. 2006) during the 4-sec study phase. In addition, the saccades between the two AOIs were analyzed. The saccades referred to the eye movements between the two AOIs that were larger than 2 degrees and with the movement speeds greater than 35 degrees per second (Liu et al. 2017; Smith and Squire 2017). ANOVAs with encoding condition (similar, different) and picture type (target, lure) as factors were performed for each parameter during encoding. One participant's eyetracking data were not recorded, so data from only 17 participants were analyzed.

To explore whether the eye-movements predicted the subsequent memory performance (i.e., difference in memory, Dm), we sorted the target pictures as "remembered" versus "forgotten" for each condition and analyzed whether this Dm effect was reflected in the percentage of fixation time and number of saccades. The Dm effect was calculated for the tasks of 2AFC, similar/different judgment and left/right judgment. ANOVAs of encoding condition (similar, different), retention interval and picture type (target, lure) were performed for each memory task for the percentage of fixation time. We also analyzed the Dm effects for other eye-movement parameters (i.e., the number of fixations, the fixation time, percentage of the number of fixations), and their results were similar to that of the percentage of fixation time, so only the latter was reported. The Dm effect for the number of saccades with the retention interval and encoding condition as factors was also analyzed (two-tailed, $P<0.05$ ).

\section{Acknowledgments}

This research was supported by the grant from the National Natural Science Foundation of China (31571114, J.Y.). The funder had no role in study design, data collection and analysis, decision to publish, or preparation of the manuscript.

\section{References}

Andermane N, Bowers JS. 2015. Detailed and gist-like visual memories are forgotten at similar rates over the course of a week. Psychon Bull Rev 22: 1358-1363. 
Brady TF, Konkle T, Alvarez GA, Oliva A. 2008. Visual long-term memory has a massive storage capacity for object details. Proc Natl Acad Sci 105: 14325-14329.

Buchler NG, Light LL, Reder LM. 2008. Memory for items and associations: distinct representations and processes in associative recognition. J Mem Lang 59: 183-199.

Calvo MG, Lang PJ. 2004. Gaze patterns when looking at emotional pictures: motivationally biased attention. Motiv Emot 28: 221-243.

Carvalho PF, Goldstone RL. 2014. Effects of interleaved and blocked study on delayed test of category learning generalization. Front Psychol 5: 936.

Christianson SA, Loftus EF, Hoffman H, Loftus GR. 1991. Eye fixations and memory for emotional events. J Exp Psychol Learn Mem Cogn 17: 693-701.

Chua HF, Boland JE, Nisbett RE. 2005. Cultural variation in eye movements during scene perception. Proc Natl Acad Sci 102: 12629-12633.

Cohen NJ, Eichenbaum H. 1993. Memory, amnesia, and the hippocampal system. The MIT Press, Cambridge, MA.

Craik FIM, Lockhart RS. 1972. Levels of processing-framework for memory research. J Verbal Learn Verbal Behav 11: 671-684.

Czerniawski J, Guzowski JF. 2014. Acute neuroinflammation impairs context discrimination memory and disrupts pattern separation processes in hippocampus. J Neurosci 34: 12470-12480.

Davachi L, Mitchell JP, Wagner AD. 2003. Multiple routes to memory: distinct medial temporal lobe processes build item and source memories. Proc Natl Acad Sci 100: 2157-2162.

Eichenbaum H, Otto T, Cohen NJ. 1994. 2 functional components of the hippocampal memory system. Behav Brain Sci 17: 449-472.

Eichenbaum H, Yonelinas AP, Ranganath C. 2007. The medial temporal lobe and recognition memory. Annu Rev Neurosci 30: 123-152.

Frankland PW, Cestari V, Filipkowski RK, McDonald RJ, Silva AJ. 1998. The dorsal hippocampus is essential for context discrimination but not for contextual conditioning. Behav Neurosci 112: $863-874$.

Gardiner JM, Java RI. 1991. Forgetting in recognition memory with and without recollective experience. Mem Cognit 19: 617-623.

Good M, Honey RC. 1991. Conditioning and contextual retrieval in hippocampal rats. Behav Neurosci 105: 499-509.

Hannula DE, Althoff RR, Warren DE, Riggs L, Cohen NJ, Ryan JD. 2010. Worth a glance: using eye movements to investigate the cognitive neuroscience of memory. Front Hum Neurosci 4: 166.

Hannula DE, Baym CL, Warren DE, Cohen NJ. 2012. The eyes know: eye movements as a veridical index of memory. Psychol Sci 23: 278-287.

Henderson JM. 2003. Human gaze control during real-world scene perception. Trends Cogn Sci 7: 498-504.

Henderson JM, Hollingworth A. 2003. Eye movements and visual memory: detecting changes to saccade targets in scenes. Percept Psychophys 65: 58-71.

Hockley WE, Consoli A. 1999. Familiarity and recollection in item and associative recognition. Mem Cognit 27: 657-664.

Holdstock JS, Mayes AR, Roberts N, Cezayirli E, Isaac CL, O'Reilly RC, Norman KA. 2002. Under what conditions is recognition spared relative to recall after selective hippocampal damage in humans? Hippocampus 12: $341-351$

Hollingworth A, Richard AM, Luck SJ. 2008. Understanding the function of visual short-term memory: transsaccadic memory, object correspondence, and gaze correction. J Exp Psychol Gen 137: 163-181.

Huebner GM, Gegenfurtner KR. 2012. Conceptual and visual features contribute to visual memory for natural images. PLos One 7: e37575.

Irwin DE. 1991. Information integration across saccadic eye-movements. Cogn Psychol 23: 420-456.

Koutstaal W, Schacter DL, Galluccio L, Stofer KA. 1999. Reducing gist-based false recognition in older adults: encoding and retrieval manipulations. Psychol Aging 14: 220-237.

Kowler E. 2011. Eye movements: the past 25 years. Vision Res 51: $1457-1483$.

Liu Z-X, Shen K, Olsen RK, Ryan JD. 2017. Visual sampling predicts hippocampal activity. J Neurosci 37: 599-609.

Lockhart RS, Craik FIM. 1990. Levels of processing - a retrospective commentary on a framework for memory research. Can J Psychol 44: 87-112.
Mayes A, Montaldi D, Migo E. 2007. Associative memory and the medial temporal lobes. Trends Cogn Sci 11: 126-135.

McClelland JL, McNaughton BL, O'Reilly RC. 1995. Why there are complementary learning-systems in the hippocampus and neocortexinsights from the successes and failures of connectionist models of learning and memory. Psychol Rev 102: 419-457.

McHugh TJ, Jones MW, Quinn JJ, Balthasar N, Coppari R, Elmquist JK, Lowell BB, Fanselow MS, Wilson MA, Tonegawa S. 2007. Dentate gyrus NMDA receptors mediate rapid pattern separation in the hippocampal network. Science 317: 94-99.

Melcher D, Colby CL. 2008. Trans-saccadic perception. Trends Cogn Sci 12: $466-473$.

Migo E, Montaldi D, Norman KA, Quamme J, Mayes A. 2009. The contribution of familiarity to recognition memory is a function of test format when using similar foils. Q J Exp Psychol 62: 1198-1215.

Moscovitch M, Craik FIM. 1976. Depth of processing, retrieval cues, and uniqueness of encoding as factors in recall. J Verbal Learn Verbal Behav 15: $447-458$.

Moscovitch M, Cabeza R, Winocur G, Nadel L. 2016. Episodic memory and beyond: the hippocampus and neocortex in transformation. Annu Rev Psychol 67: 105-134.

Niibori Y, Yu T-S, Epp JR, Akers KG, Josselyn SA, Frankland PW. 2012. Suppression of adult neurogenesis impairs population coding of similar contexts in hippocampal CA3 region. Nat Commun 3: 1253.

Norman KA, O'Reilly RC. 2003. Modeling hippocampal and neocortical contributions to recognition memory: a complementary-learning-systems approach. Psychol Rev 110: 611-646.

Nummenmaa L, Hyona J, Calvo MG. 2006. Eye movement assessment of selective attentional capture by emotional pictures. Emotion 6: 257-268.

Ranganath C, Yonelinas AP, Cohen MX, Dy CJ, Tom SM, D'Esposito M. 2004. Dissociable correlates of recollection and familiarity within the medial temporal lobes. Neuropsychologia 42: 2-13.

Rayner K. 1998. Eye movements in reading and information processing: 20 years of research. Psychol Bull 124: $372-422$.

Rayner K. 2009. Eye movements and attention in reading, scene perception, and visual search. Q J Exp Psychol 62: 1457-1506.

Ryan JD, Althoff RR, Whitlow S, Cohen NJ. 2000. Amnesia is a deficit in relational memory. Psychol Sci 11: 454-461.

Ryan JD, Hannula DE, Cohen NJ. 2007. The obligatory effects of memory on eye movements. Memory 15: 508-525.

Sadeh T, Ozubko JD, Winocur G, Moscovitch M. 2014. How we forget may depend on how we remember. Trends Cogn Sci 18: 26-36.

Sahay A, Scobie KN, Hill AS, O'Carroll CM, Kheirbek MA, Burghardt NS, Fenton AA, Dranovsky A, Hen R. 2011. Increasing adult hippocampal neurogenesis is sufficient to improve pattern separation. Nature 472: 466-470.

Sharot T, Davidson ML, Carson MM, Phelps EA. 2008. Eye movements predict recollective experience. PLos One 3: e2884.

Slamecka NJ, McElree B. 1983. Normal forgetting of verbal lists as a function of their degree of learning. J Exp Psychol Learn Mem Cogn 9: 384-397.

Smith CN, Squire LR. 2017. When eye movements express memory for old and new scenes in the absence of awareness and independent of hippocampus. Learn Mem 24: 95-103.

Voss JL, Warren DE, Gonsalves BD, Federmeier KD, Tranel D, Cohen NJ. 2011. Spontaneous revisitation during visual exploration as a link among strategic behavior, learning, and the hippocampus. Proc Natl Acad Sci 108: E402-E409.

Wang S-H, Teixeira CM, Wheeler AL, Frankland PW. 2009. The precision of remote context memories does not require the hippocampus. Nat Neurosci 12: 253-255.

Wiltgen BJ, Zhou M, Cai Y, Balaji J, Karlsson MG, Parivash SN, Li W, Silvaz AJ. 2010. The hippocampus plays a selective role in the retrieval of detailed contextual memories. Curr Biol 20: 1336-1344.

Received March 1, 2018; accepted in revised form September 11, 2018. 


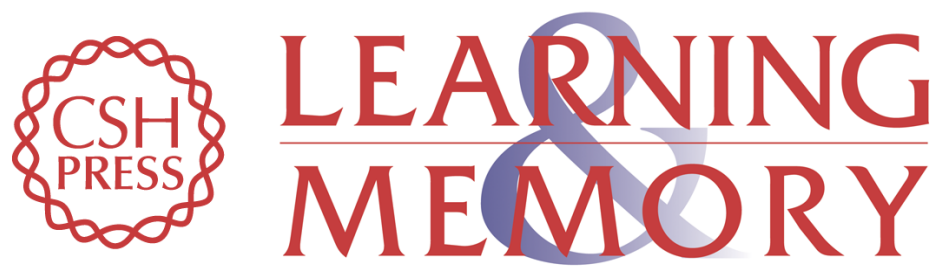

\section{Discriminative learning of similar objects enhances memory for the objects and contexts}

Wenxi Zhou, Haoyu Chen and Jiongjiong Yang

Learn. Mem. 2018, 25:

Access the most recent version at doi:10.1101/Im.047514.118

References This article cites 51 articles, 8 of which can be accessed free at:

http://learnmem.cshlp.org/content/25/12/601.full.html\#ref-list-1

Creative This article is distributed exclusively by Cold Spring Harbor Laboratory Press for the

Commons

first 12 months after the full-issue publication date (see

License http://learnmem.cshlp.org/site/misc/terms.xhtml). After 12 months, it is available under a Creative Commons License (Attribution-NonCommercial 4.0 International), as described at http://creativecommons.org/licenses/by-nc/4.0/.

Email Alerting Receive free email alerts when new articles cite this article - sign up in the box at the Service top right corner of the article or click here. 